Research Article

\title{
CLASSIFICATION OF APPLE LEAF DISEASES USING CONVOLUTION NEURAL NETWORK APPROACH
}

\section{Halit ÇETINER*}

Isparta University of Applied Science, Vocational School of Technical Sciences, Computer Technology, Isparta, Turkey

\begin{tabular}{ll}
\hline Keywords & Abstract \\
\hline $\begin{array}{l}\text { Convolutional Neural } \\
\text { Network, }\end{array}$ & $\begin{array}{l}\text { It is difficult to constantly control apple trees in farmland. In case of a disease on } \\
\text { tree leaves, the risk of disease transmission to other leaves is high. It is necessary to } \\
\text { Apple leaf, }\end{array}$ \\
SMOTE, & $\begin{array}{l}\text { prevent further deterioration of the plant by performing automatic detection of the } \\
\text { disease in the early period. If the disease detection is delayed, the planned } \\
\text { Pre Trained Network. }\end{array}$ \\
& production cannot be realized. It is too late if diseases are detected by a farmer or \\
& agronomist. In addition, as the agricultural lands grow, the number of experts \\
& needed increases accordingly. For these reasons, leaf images of apple trees are \\
grouped into 4 different classes: apple peel, leaf rust, healthy apple and multiple \\
disease states. In the proposed method, noise removal in the images, detection of \\
the relevant area and histogram equalization on the YUV color space are performed. \\
Due to the unbalanced class distribution in the data set used, data augmentation was \\
applied for the minority classes with the SMOTE method. Afterwards, features are \\
extracted using pre-trained network models DenseNet121, DenseNet201, \\
InceptionResNetV2, InceptionV3, ResNet50V2. Extracted features were classified \\
with a CNN-based method developed with an accuracy of 99\%.
\end{tabular}

\section{KONVOLÜSYON SİNİR AĞI YAKLAŞIMI KULLANARAK ELMA YAPRAĞI HASTALIKLARININ SINIFLANDIRILMASI}

\begin{tabular}{|c|c|}
\hline Anahtar Kelimeler & Öz \\
\hline $\begin{array}{l}\text { Konvolüsyonel Sinir Ağı, } \\
\text { Görüntü İssleme, } \\
\text { Elma Yapră̆ı, } \\
\text { SMOTE, } \\
\text { Önceden Eğitilmiş Ağ }\end{array}$ & $\begin{array}{l}\text { Tarım arazilerindeki elma ağaçlarını sürekli olarak kontrol etmek zordur. Ağaç } \\
\text { yapraklarında oluşan bir hastalık durumunda diğer yapraklara hastalı̆̆ın bulaş riski } \\
\text { yüksektir. Erken dönemde hastalığın otomatik tespitini gerçekleştirerek bitkinin } \\
\text { daha fazla bozulmasını önlemek gereklidir. Eğer hastalık tespitinde geç kalınırsa } \\
\text { planlanan üretim gerçekleştirilememektedir. Bir çiftçi ya da tarım uzmanı } \\
\text { tarafından hastalıkların tespit edilmesi durumunda geç kalınmaktadır. Buna ek } \\
\text { olarak tarım arazileri büyüdükçe ihtiyaç duyulan uzman sayısı da ona göre artış } \\
\text { göstermektedir. Bu sebeplerden dolayı elma ağaçlarına ait yaprak görüntülerini } \\
\text { kullanarak ağaç yaprakları elma kabuğu, yaprak pası, sağlıklı elma ve birden fazla } \\
\text { hastalık durumları olmak üzere } 4 \text { farklı sınıfa gruplandırılmıştır. Öne sürülen } \\
\text { yöntemde görüntülerde gürültülerin temizlenmesi, ilgili alanın tespiti ve YUV renk } \\
\text { uzayı üzerinde histogram eşitleme gerçekleştirilmiştir. Kullanılan veri setinde sınıf } \\
\text { dağllımlarının dengesiz olmasından dolayı SMOTE yöntemi ile azınlık olarak kalan } \\
\text { sınıflar için veri büyütmesi uygulanmıştır. Sonrasında DenseNet121, DenseNet201, } \\
\text { InceptionResNetV2, InceptionV3, ResNet50V2 önceden eğitilmiş ağ modelleri } \\
\text { kullanılarak öznitelikler çıkartılmıştır. Çıkartılan öznitelikler geliştirilen CNN } \\
\text { tabanlı bir yöntemle } 99 \% \text { doğruluk oranında snıflandırılma gerçeklestirilmistir. }\end{array}$ \\
\hline
\end{tabular}

Alıntı / Cite

Çetiner, H., (2021). Classification of Apple Leaf Diseases Using Convolution Neural Network Approach, Journal of Engineering Sciences and Design, 9(4), 1130-1140.

\footnotetext{
* ilgili yazar / Corresponding author: halitcetiner@isparta.edu.tr, +90-246-214-6935
} 


\begin{tabular}{l|l|l}
\hline Yazar Kimliği / Author ID (ORCID Number) & \multicolumn{3}{|l}{ Makale Süreci / Article Process } \\
\hline H. Çetiner, 0000-0001-7794-2555 & Başvuru Tarihi / Submission Date & 09.08 .2021 \\
& Revizyon Tarihi / Revision Date & 09.09 .2021 \\
& Kabul Tarihi / Accepted Date & 12.09 .2021 \\
& Yayım Tarihi / Published Date & 20.12 .2021 \\
\hline
\end{tabular}

\section{Introduction}

Due to different environmental factors, fruit production undergoes great changes. The decrease in production as a result of various effects causes the balance in the agricultural industry to deteriorate. Apple is a durable fruit that can be grown in many different cities in Turkey, especially in Kayseri and Isparta and can be preserved for a long time. On the world scale, it is the most produced fruit type after banana and watermelon in 2018. More than approximately 86 million tons were produced (Sottocornola et al., 2021). These figures are underestimated relative to the potential apple yield. Among the main reasons for this are the fact that the farmers engaged in agricultural production do not use technology in production, and that quality planting materials are not purchased due to infections, insect pests and economic restrictions. It is seen that more than half of Chinese producers use the internet in order to increase efficiency in apple production. It has been determined that the productivity in apple production using the Internet has increased by at least 30\% (Zhu et al., 2021). It is seen that the Chinese government supports the agricultural information model called Internet Plus Agriculture Model in order to provide rural and agricultural development (Han et al., 2021; Ni et al., 2021). It is noted that with these uses, the vacant agricultural lands are evaluated, there is a great increase in land rentals and as a result of these, there is an increase in the number of labor force (Deng et al., 2019; Hou et al., 2019).

Apples can be processed industrially in different functional forms such as juice and pulp. However, it is seen as a source of bioactive components that enhance the immune system (Duralija et al., 2021). In addition to the many benefits of apple fruit on humans, it is prone to diseases caused by different microorganisms or bacteria. In addition to increasing employment opportunities, agricultural products that provide food and income lose $42 \%$ of the world's total by various microorganisms every year (Tahir et al., 2021). Fertilizing trees at certain times is seen as an important step in order to protect them from diseases. If the leaves change despite the fertilization process, the system can help the farmer to diagnose. In this way, it is desired to prevent the farmer from experiencing too much loss. Farmers who do not use any technique or technology miss the appropriate time to protect trees from diseases. Incorrect or untimely treatment ensures the rapid spread of leaf diseases. Insect damage and diseases are increasing, especially because pathogen-type viruses multiply in a short time. In addition to these, environmental damages and costs increase due to excessive or insufficient use of chemicals in case of possible misdiagnosis (Thapa et al., 2020).

Diagnosing the disease with the classical methods we have seen from our ancestors increases the processing time. Even in today's conditions, the diagnosis of the disease is often made by a specialist supervisor. In agricultural lands with thousands of trees, this situation requires a lot of experts. As a result, the lack of automatic systems inevitably leads to a decrease in fruit quality. When the planned number of products cannot be produced, great losses are experienced in terms of economy and time. Today, with the development of technology, it is seen that auxiliary tools have been created for farmers. Recently, pre-network-based methods and image processing algorithms are frequently used in the classification and automatic detection of leaf diseases of various plants (Gargade \& Khandekar, 2019; Gobalakrishnan et al., 2020; Shrivastava, 2021; Tahir et al., 2021). When the studies are looked back, it is seen that mostly classical machine learning algorithm methods are used (Annabel et al., 2019; Gobalakrishnan et al., 2020; Shrivastava, 2021). It has been determined that there are different efforts to classify different diseases that occur in various plants in order to provide convenience to the farmers and to support the country's economy. In one of them; Sujatha et al. mention that they use machine learning-based methods to diagnose foliar diseases of different citrus plants such as lemons (Sujatha et al., 2021). In another, an automated system has been proposed for diagnosing foliar diseases of maize and potato plants (Aurangzeb et al., 2020). In another study, Yun et al. tried to classify the leaf images of the cucumber plant with probability-based neural networks (Shi et al., 2015). Iqbal et al. examines the process steps of existing studies in the literature that classify diseases in citrus plants such as oranges and lemons. As a result of the investigations, Iqbal et al. are reported that studies on the diagnosis and classification of diseases in plants are at the initial stage (Iqbal et al., 2018). Looking at the current studies, it tries to determine the texture, size, color status and shape of the fruit or leaf where deterioration occurs. In addition, it diagnoses the disease by segmenting the stem, leaves and fruit. In order to obtain stable results in classical image processing methods, there should be a large number of training examples (Khan et al., 2020). Such methods can also have various problems such as light scattering, brightness, contrast irregularities, similarities between the defective lesion area and the normal area. Recently, these problems have been dealt with effectively with pre-trained neural network methods created with thousands of classes in very different categories to overcome all these problems. 
The main contribution to this article study is given below in articles:

- A useful tool has been developed for farmers. Performance analyzes of pre-trained deep neural networks popular in the literature in recent years have been made.

- As a result of the analysis, the system, which will be based on pre-trained feature extraction, image processing and convolution neural networks (CNN), is expected to relieve the farmer in some issues.

- Attributes were obtained from pre-processed plant disease images with the help of DenseNet121, DenseNet201, InceptionResNetV2, InceptionV3, ResNet50V2 pre-trained network models. Performance levels were measured to determine which of the methods used was good at identifying the type of leaf disease.

- With a proposed basic CNN structure model, a structure model was created to predict test images. In this way, when a new test image is given to the system created, the type of apple leaf disease is estimated.

- It will support the increase of apple production in agricultural lands with the spread of early diagnosis in the agricultural sector.

The next sections of the article are planned as follows. In the Related Studies section, articles that are close to our work in the previous literature were examined. In the section called Proposed Method, the steps of the developed algorithm are mentioned. In the Results and Discussion section, the experimental results using the proposed model are explained by comparing them with the studies using the same data set. The study was concluded in the section titled Conclusions.

\section{Related Work}

When existing studies in the literature were analyzed, plant diseases were determined by using attributes such as color, texture, shape and shape of plant lesions (Prashar et al., 2017; Singh \& Misra, 2017; Tiwari et al., 2020). Inspired by these studies, some preprocessing was done on leaf images before they were given as input to deep learning algorithms. The main purpose of preprocessing is to reduce the image size and perform basic operations in the ROI region. Different color spaces were examined by researching the color spaces in the ROI region. Distribution was adjusted using histogram equalization in regions where unbalanced color distributions were detected. Liang et al. classified leaf disease with a RESNET-based deep learning method (Liang et al., 2019). Zhang et al. created a neural network with three different convolutions using RGB color channels. Classified vegetable leaf diseases using this neural network model (Zhang et al., 2019). Türkoğlu et al. developed a neural network that classifies the features obtained by using some layers of pre-trained network models such as DenseNet201, GoogleNet, AlexNet, using the LSTM method (Turkoglu et al., 2019). Performance analyzes of the methods used were used for apple disease classification. Wang et al. created a structure that automatically predicts the severity of the disease in order to measure the yield loss due to the disease (Wang et al., 2017). He states that this structure provides the best results with the VGG16 pre-trained network model. According to Dubey et al. worked to classify apple diseases using different image attributes such as shape, texture, and color (Dubey \& Jalal, 2016). With the help of the K-means algorithm, the lesion area was determined. Afterwards, he defined and classified the statistical features that define that region. Ferentinos developed a CNN model to group a dataset of healthy and diseased images of different plants (Ferentinos, 2018). Ferentinos mentions the difficulty of observing the signs of plant diseases optically. Even by agronomists and plant pathologists, it is often mentioned that they fail to determine the type, type of some diseases. It is emphasized that it will provide great convenience to expert agricultural engineers who make automatic detection and diagnosis of plant diseases by optical methods (Mohanty et al., 2016). When the studies are examined, it is stated that it is difficult to detect and classify leaf diseases classically by agricultural engineers and plant pathologists working in the field.

\section{Proposed Method}

\subsection{Material}

In this study, an apple leaf dataset with varying levels of illumination angle and surface and noisy features was used. The dataset used includes one healthy class and three different apple leaf disease classes. A total of 3642 leaf images were used. This data set was created in order to prevent yield loss due to different problems such as stress in apple orchards in the USA. It is reported that the generated data set is complex. It is an unequal data set that includes different types of diseases. They are not data sets with the same backgrounds taken in special environments. There are non-homogeneous leaf background images. Images were taken regularly at different times of the day to increase the difficulties in the lighting situation. Five percent of the data set has both crusting disease and rust disease (Thapa et al., 2020). The features mentioned in the dataset affect the detection accuracy of the leaf disease analysis. In the data set used, there were 516 apple leaf images in the healthy class, 91 in the multiple diseases (MD) class, 622 in the rust class, and 592 in the scab class. There are 1821 educational leaf images in total. There are also the same number of test images. The Healthy class shown in Figure 1 does not have 
any signs of disease. It represents healthy leaves consisting of pure green without spots and markings. Likewise, MD is a disease group that has both crusting and rusting disease at the same time. Rust is a group of diseases caused by rust fungus with yellow shapes on the leaf. The last group of diseases, Scab, is a group of diseases consisting of fungus with brown wounds and scars.

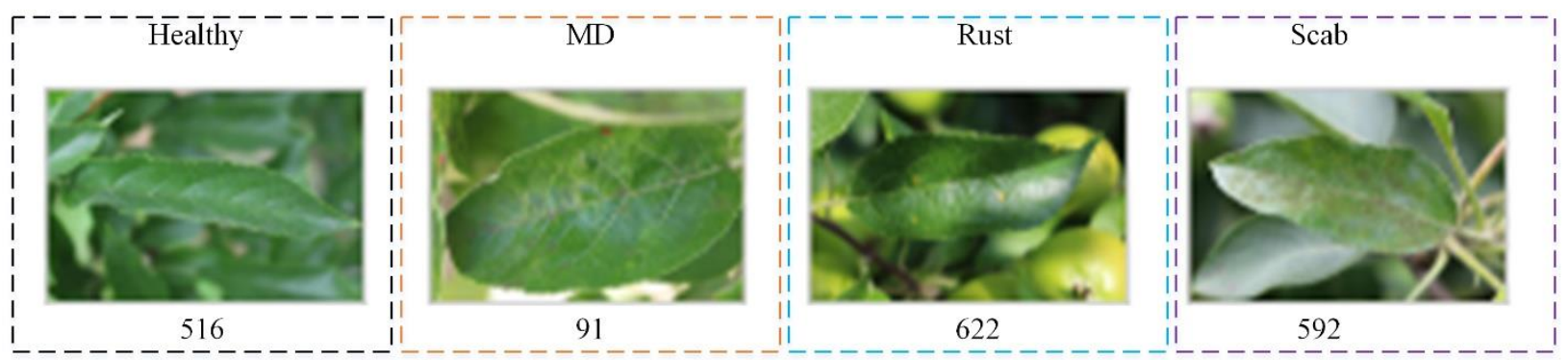

Figure 1. Sample images from the classes in the dataset and their total number

\subsection{Proposed Feature Extraction Steps (PFEM)}

Deep learning is defined as a cluster under the domain of machine learning that enables learning by extracting important attributes from complex or normal datasets with minimal preprocessing. Compared to classical machine learning methods, deep learning methods can be used without any preprocessing. However, in this study, preprocessing applications were deemed necessary due to structures that may affect the classification result such as noise in the images. The feature extraction step, which exists in classical machine learning algorithms, can be optionally performed in deep learning algorithms. In both algorithms, the steps of image sizing, noise removal, edge detection, histogram equalization, ROI region determination are common.

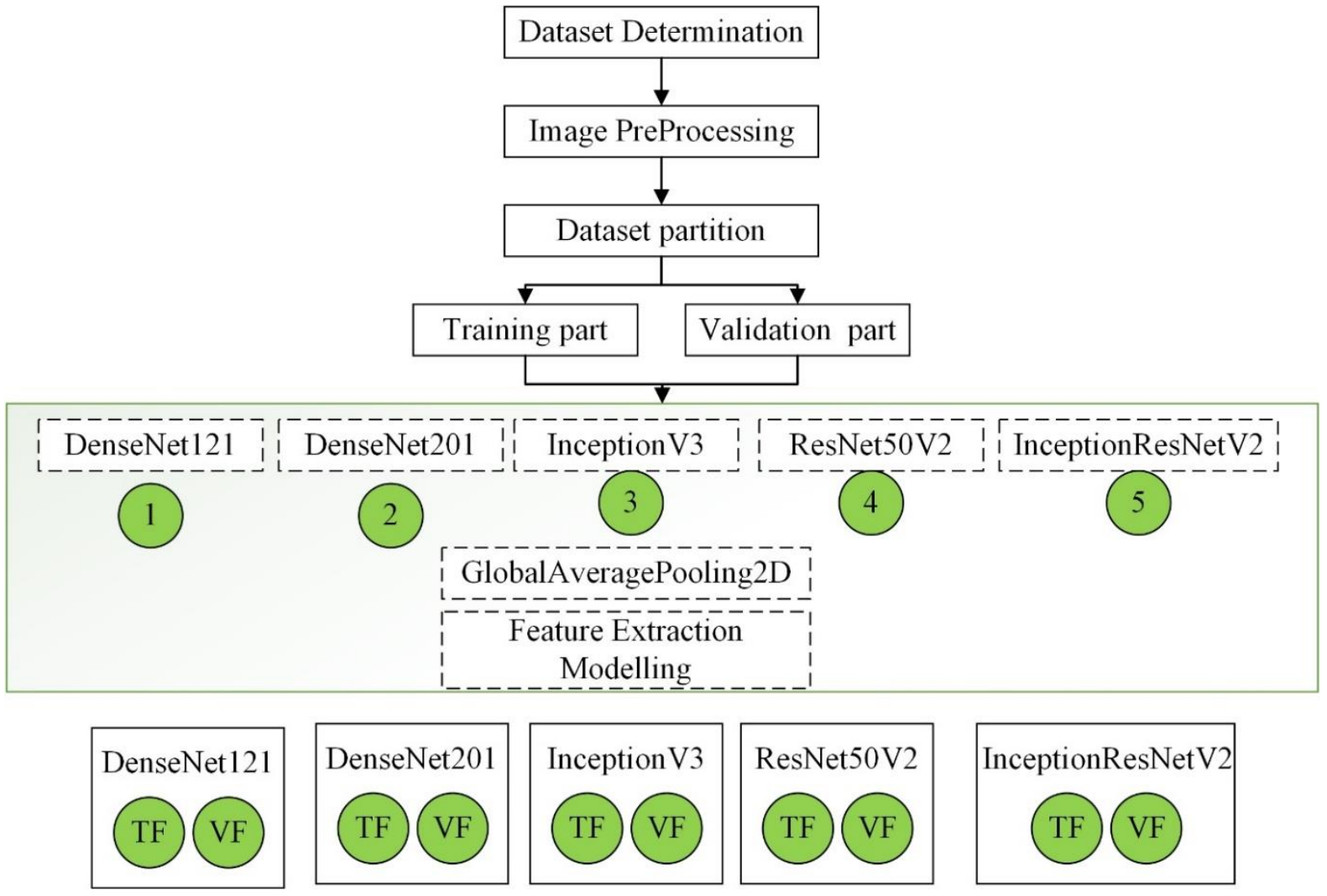

Figure 2. Proposed Feature Extraction Steps (PFEM)

$$
\begin{aligned}
& \hat{u}_{i}(p)=\frac{1}{C(p)} \sum_{q \in B(p, r)} u_{i}(q) w(p, q), C(p)=\sum_{q \in B(p, r)} w(p, q) \\
& d^{2}(B(p, f), B(q, f))=\frac{1}{3(2 f+1)^{2}} \sum_{i=1}^{3} \sum_{j \in B(0, f)}\left(u_{i}(p+j)-u_{i}(q+j)\right)^{2}
\end{aligned}
$$


The steps shown in Figure 2 illustrate the proposed feature extraction model (PFEM) steps. In the Dataset Determination step, a data set with an non-uniform background, varying lighting status, and more than one disease was determined by looking at the degree of difficulty. The second step of PFEM is the image preprocessing step. This step consists of the combination of different functions in itself. In the preprocessing step, all of the training images were resized according to the size $(224,224.3)$. The specified sizing will provide ease of processing during input to pre-trained network models. Afterwards, a method that provides noise filtering over the color averages of different neighborhoods of the image is used. This method is explained in Equations 1 and 2 . In Equation $1, u=$ $\left(u_{1}, u_{2}, u_{3}\right)$ shows the color channels in order to remove the noise in a color image. The $i$ value represents $1,2,3$ values, $p$ represents a specific pixel value. The pixel dimensions of $(2 r+1) \times(2 r+1)$ represent the central neighbors in $p, B(p, r)$. The value of $w(p, q)$ in the central color regions of $(2 f+1) \times(2 \mathrm{f}+1)$ in Equation 2 depends on the squared Euclidean distance named $d^{2}(B(p, f), B(q, f))$. In Figure 2, the neighborhood-based noise removal result of an image in the MD class in the data set is shown. After the noise removal step, edge detection was performed. In edge detection (Hoang \& Nguyễn Quốc, 2018), the prewitt algorithm was preferred because of its success in the literature. It can detect edges using a certain threshold value even in gray level and noisy images. In Equation $3, P_{x}$ indicates the operator applied on the horizontal axis, while $P_{y}$ indicates the operator applied on the vertical axis. Then, the gradient of the edge values obtained in both directions is calculated. The gradient formula is shown in Equation 4. YUV color space is preferred over RGB color space to better handle challenges such as light differences mentioned in the image. At the beginning of the study, all data set images were converted to red, green, blue channels, Y, U, V channels by histogram equalization according to the color distribution status. The minimum and maximum color range values of each color channel were determined.
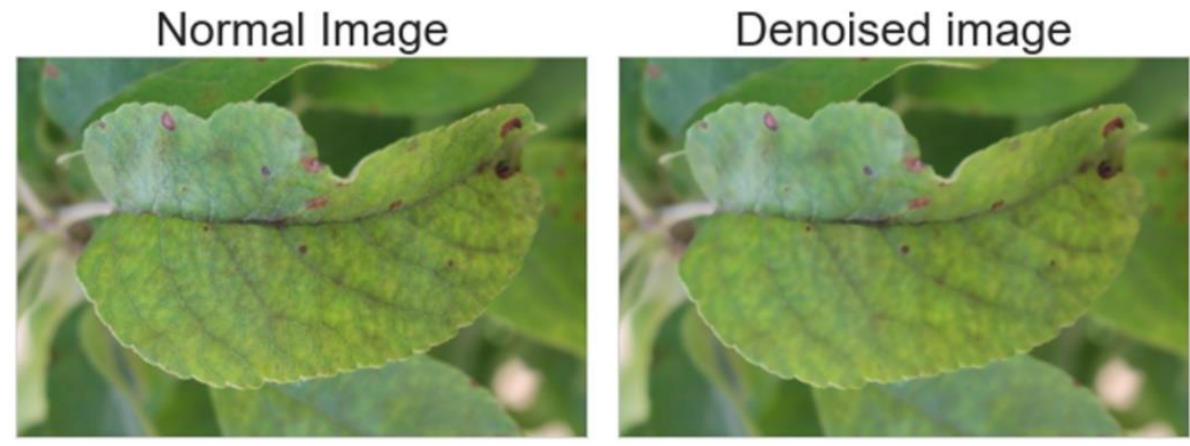

Figure 3. Denoised image

These ranges are given in Table 1. In Figure 4, the color distribution in the $U$ and $V$ channels collapsed to the right or left is seen. Histogram equalization was performed after the region of uneven color distribution and the region of ROI that should be of interest were determined.

$$
\begin{aligned}
& P_{x}=\left(\begin{array}{ccc}
-1 & -1 & -1 \\
0 & 0 & 0 \\
1 & 1 & 1
\end{array}\right) \quad P_{y}=\left(\begin{array}{ccc}
-1 & 0 & 1 \\
-1 & 0 & 1 \\
-1 & 0 & 1
\end{array}\right) \\
& P=\sqrt{\left(P_{x}\right)^{2}+\left(P_{y}\right)^{2}}
\end{aligned}
$$

Figure 5 shows the result of histogram equalization. All the steps in the preprocessing step were applied to the training images.

Table 1. YUV color channel distributions of all classes

\begin{tabular}{|c|c|c|}
\hline Color Channel & Minimum & Maximum \\
\hline $\mathrm{Y}$ & 67.12 & 162.32 \\
\hline $\mathrm{U}$ & 87.38 & 130.98 \\
\hline $\mathrm{V}$ & 94.19 & 142.55 \\
\hline
\end{tabular}



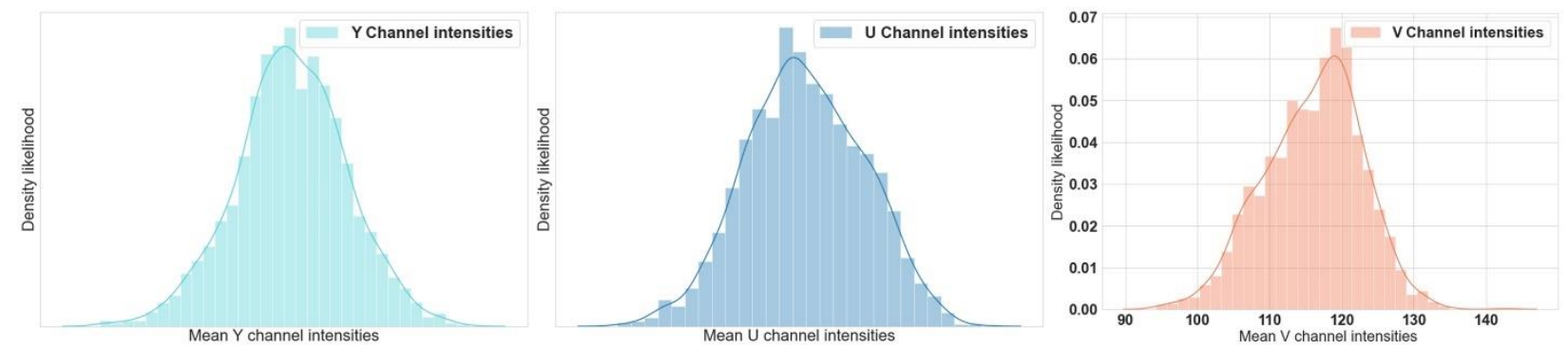

Figure 4. Uneven color distrubiton

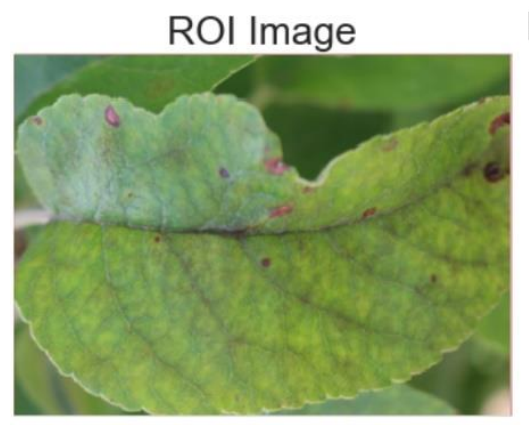

Histogram Equalized ROI Image YUV

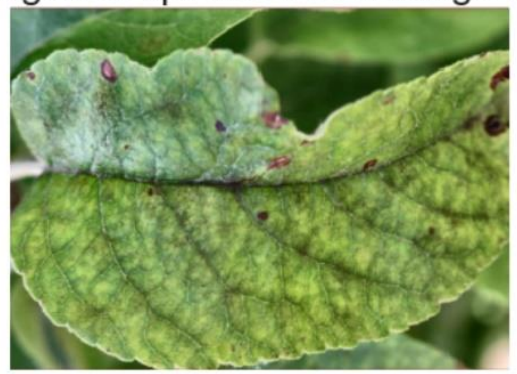

Figure 5. Histogram Equalized ROI Image YUV

In the last part of the preprocessing stage, the data were given as input to the Synthetic Minority Oversampling Technique (SMOTE) method to eliminate the irregularities in the data distributions between the classes. This technique is a method of oversampling the minority classes to match the other classes. Increases the number of data samples by using Euclidean distance with nearest neighbor methodology (Ishaq et al., 2021). Since the data is sampled based on the original data, it becomes similar to the original data. As a result of this process, 2488 preprocessed images in the YUV color space $(2488,224,224.3)$ and a set of labels indicating which class each image in (2488.4) are included in were created. The final version of the created data set is shown in Figure 6. While Healthy, MD, Rust, Scab are 516, 91, 622, 592, respectively, in the figure on the left side of Figure 6, the sample number on the right side is 622 .
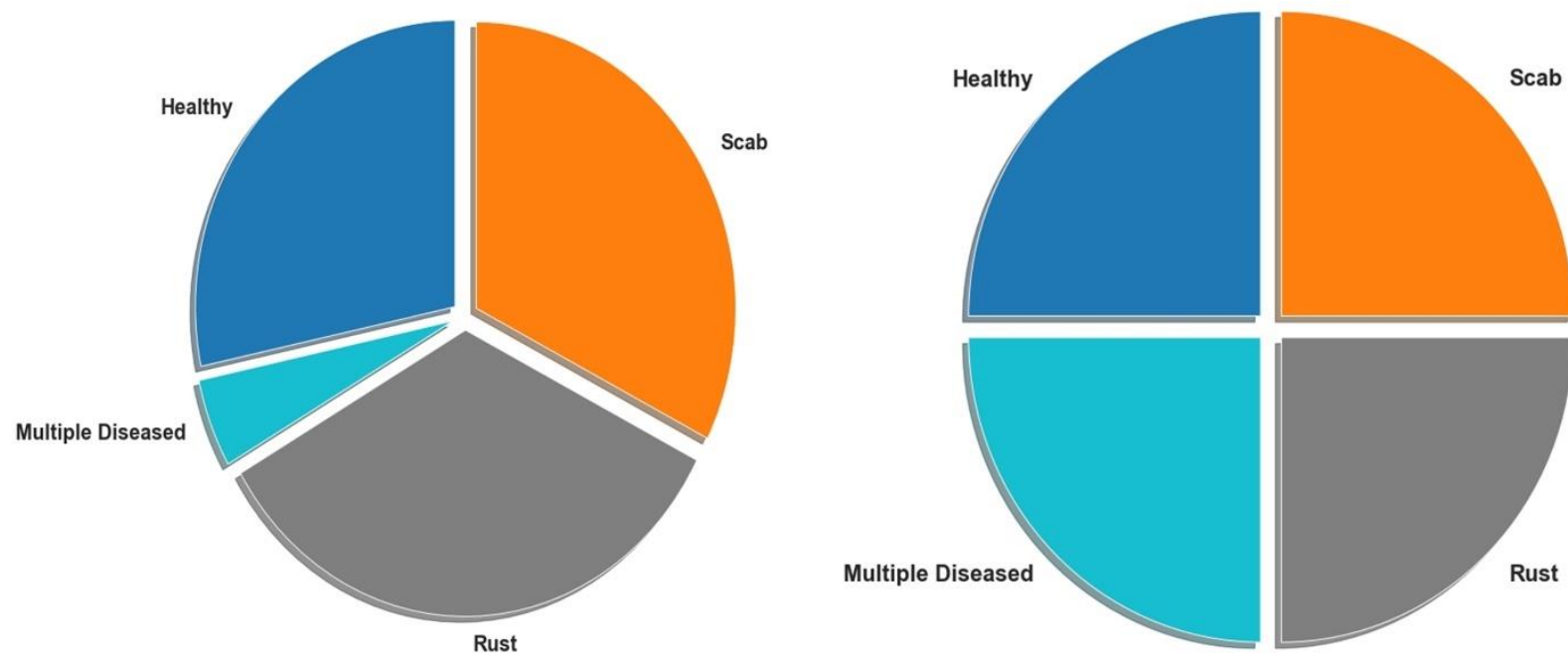

Figure 6. Data distribution before and after the smote process

After the preprocessing step, Data partition operation is performed. $90 \%$ of the training dataset was used as training data and $10 \%$ as validation data. The determination of these ratios was carried out by experimental studies. In determining these rates, attention was paid to ensure that the training and validation accuracy rates were high and the loss values were low. 
The next step consists of feature extraction and classification steps. Two different deep learning models are configured to perform the specified steps. In both models, different CNN structures are used. In classical methods, unsuccessful results can be obtained when the image differences that need to be processed are large. Convolutional neural networks, on the other hand, can be more successful because they can model by filtering the entire image on a pixel basis and combine this information with deep networks. While performing this process, it is carried out in a structure consisting of multiple layers. The first layer involves detecting the edge regions of an image and creating templates. The next layer takes simpler shapes from the image while using the templates for the foundation. In this way, it creates more templates than forms of different objects with various scales, positions, and illuminations. All templates are mapped to the input images in the final layers. The output obtained as a result of operations in the layers is the weighted average of all output values (Gollapudi, 2019). CNNs basically consist of three types of layers. These layers are the convolution layer, which has the task of filtering in order of use, the feature size-reducing pooling layer, and the fully connected layer that transforms it into a flat vector. These CNN structures have a three-dimensional layer adjustment in height, width and depth compared to classical neural networks. The neurons of the hidden layer in classical neural networks are connected only to a certain part of the neurons of the other layers. It is not connected to every neuron. The output obtained as a result of the operations is converted into a single vector. Unlike the hidden layers in classical neural networks, the hidden layers in CNN assist in feature extraction, along with the filters, the circulating convolution layer, and the repository layers that allow the maximum or average values to be taken in data reduction. Convolution layers are the layer that can combine multiple operations and create a feature map with matrix multiplications. The term stride refers to the pixel distance of each filter used. The resulting matrix was transformed into a nonlinear structure by passing through the ReLU activation function. The pooling layer is the layer that connects to the convolution layer, usually for data reduction purposes. This layer is similar to the feature selection step in classical neural networks. It provides parameter reduction, size reduction and overfitting to reduce training time. The maximum pooling technique, which selects the maximum value from among the values in each window, is the most widely used. Optimizes image feature size by making sure relevant key features are extracted from the image. The classification task is performed in the fully connected layer. Multidimensional data needs to be converted to one-dimensional form. It is fully interconnected with all the activations in the previous layers like normal neural networks.

\subsection{CNN based feature classification model}

Structures that have been pre-trained on a large data set and whose weights and architectures are preserved are called pre-trained networks. In these architectures, changes to be made in structures such as input, classification and full connected layer can be used in different datasets apart from the dataset in which the architecture is trained. Feature extraction can be performed when using a different data set than pre-trained networks. For this, it includes running all layers of the network on the new image, together with the layers that need to be changed in the pre-trained networks.

In this here;

- A pre-trained network is taken. The output layer is removed. The input layer is fine-tuned according to the data set used in this article.

- Added classifier with recommended CNN architecture to classify features of pre-trained network.

- Then, with these fine-tuning, the generated model is created with validation as well as training data.

- Finally, when untrained images are given to the model, recognition can be done easily.

CNN-based feature extraction model and classification model are shown in Figure 2 and Figure 7, respectively. In Figure 2, two different feature matrices named TF (Training Feature) and VF (Validation Feature) were obtained with each pre-trained architectural structure used in the classification PFEM building model. The models of TF and VF matrices obtained from each pre-trained network are used in the proposed CNN-based classification model. The pre-trained DenseNet121, DenseNet201, InceptionV3, ResNet50V2 and InceptionResNetV2 CNN algorithms used in this article generally include input layer, pooling layer, convolution layer with different filters, fully connected layer, dropout, ReLU activation layer and classification layers. 
The input layer is the structure where the images in the data set are given to the network after different preprocessing. In this layer, the input size needs to be set beforehand. Throughout this study, the inputs $(224,224)$ are set in width and height dimension. YUV color channel is used as color channel. Basically, images are preprocessed without providing input. The convolution layer is the basic layer that creates the feature matrix obtained by using different filters in the image. This layer transforms certain filters on the image. Filters can be defined in different sizes. Examples of these most commonly used dimensions are $3 \times 3,5 \times 5,7 \times 7,9 \times 9$ or $11 \times 11$. The Rectified linear units (ReLU) layer is actually the activation layer. The activation layer generally comes after the filtering operations in the image. It is used in linear and nonlinear transformation operations. It brings the actual data into a range and format that the network can understand. The pooling layer is then connected to the convolution layer, which consists of filtering operations and ReLU activation. It tries to reduce the image size without touching the memory. The full connected layer is used before the classification layer by connecting with previous neurons. It is in full connection with the feature extraction neurons before this layer. The reduction layer deletes some neurons in the network to avoid over-learning. Some randomly selected neurons can be neglected. The classification layer, as the name suggests, provides class labels based on classification inputs. It uses the softmax function to obtain class labels. The detailed properties of the pre-trained networks providing feature extraction in this study are given in Table 2 .

Table 2. Network details used in feature extraction

\begin{tabular}{|c|c|c|c|c|c|}
\hline Comparison model & Size (MB) & Parameter & Depth & $\begin{array}{c}\text { Input } \\
\text { Size }\end{array}$ & Reference \\
\hline DenseNet121 & 33 & 8.062 .504 & 121 & 224,224 & (Huang et al., 2017) \\
\hline DenseNet201 & 77 & 20.242 .984 & 201 & 224,224 & (Huang et al., 2017) \\
\hline InceptionV3 & 92 & 23.851 .784 & 159 & 224,224 & (Szegedy et al., 2016) \\
\hline ResNet50V2 & 98 & 25.613 .800 & - & 224,224 & (He et al., 2016) \\
\hline InceptionResNetV2 & 215 & 55.873 .736 & 572 & 224,224 & (Szegedy et al., 2017) \\
\hline
\end{tabular}

In Figure 7, after the leaf images pass through the PFEM steps, feature extraction is performed with the help of pre-trained networks. The first part with the leaf strings in Figure 7 represents the part of the PFEM steps up to the preprocessing stage. DenseNet121, DenseNet201, InceptionV3, ResNet50V2 and InceptionResNetV2 are given separately to the CNN classification model, and two different matrices, TV and VF, obtained separately from each of them, are given to the CNN model. With the full connected layer, each neuron is connected with the entire feature map. These links determine class scores based on the Softmax activation function. Classification layer is specialized for classifier task.

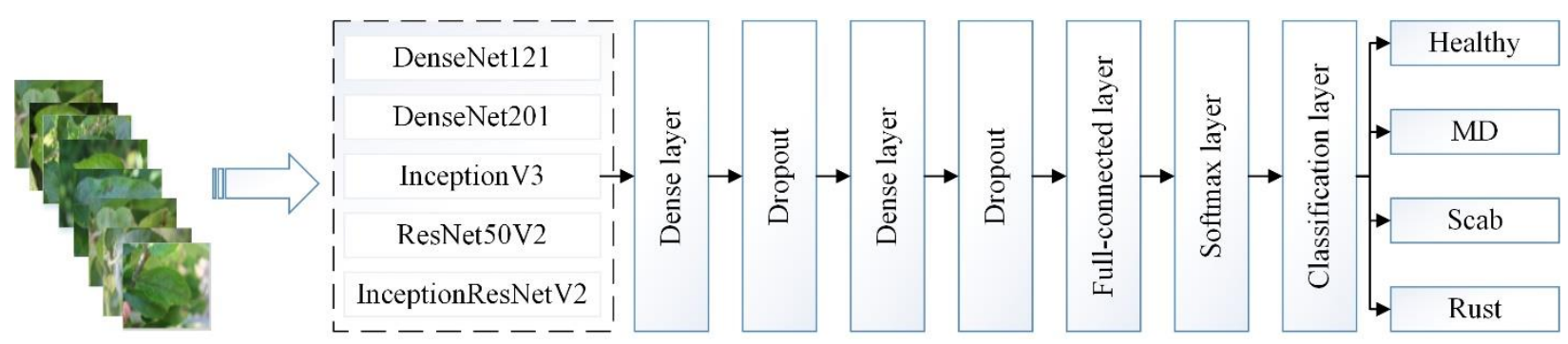

Figure 7. The proposed CNN-based feature classification model (PCNN)

\section{Results and Discussion}

All values such as epoch values are adjusted to be the same for all pre-trained networks used. As observed in Table 4, the proposed CNN algorithm from the features obtained with the ResNet50V2 network, according to the specified adjustments, gave the highest accuracy value as a result of the classification. 
Table 3. Classification results

\begin{tabular}{|l|l|l|l|l|}
\hline PFEM Model & Training Accuracy & Validation Accuracy & F1 Score & Precision \\
\hline DenseNet121 & 0.96 & 0.95 & 0.95 & 0.96 \\
\hline DenseNet201 & 0.97 & 0.98 & 0.97 & 0.98 \\
\hline InceptionV3 & 0.93 & 0.92 & 0.92 & 0.91 \\
\hline ResNet50V2 & 0.99 & 0.97 & 0.97 & 0.96 \\
\hline InceptionResNetV2 & 0.89 & 0.91 & 0.90 & 0.91 \\
\hline
\end{tabular}

a)
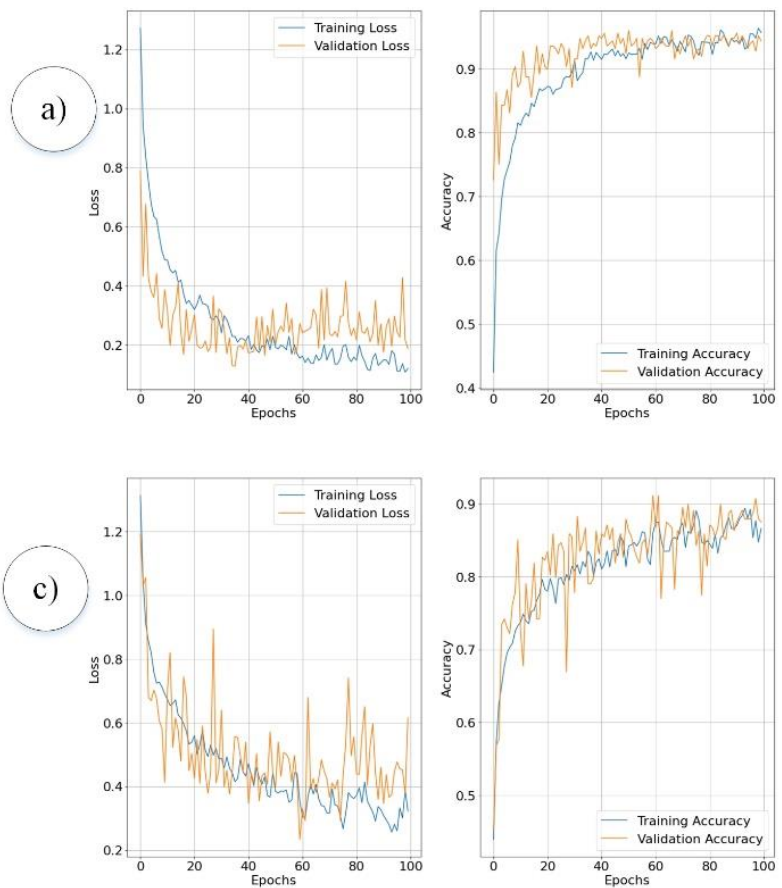

e)

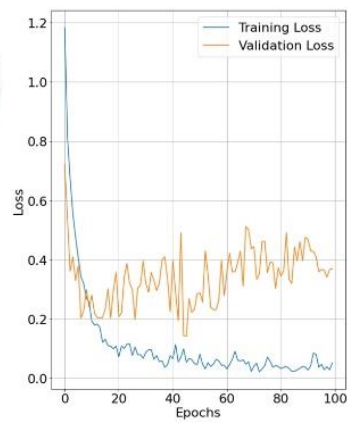

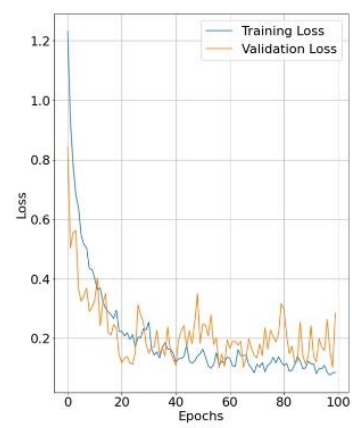
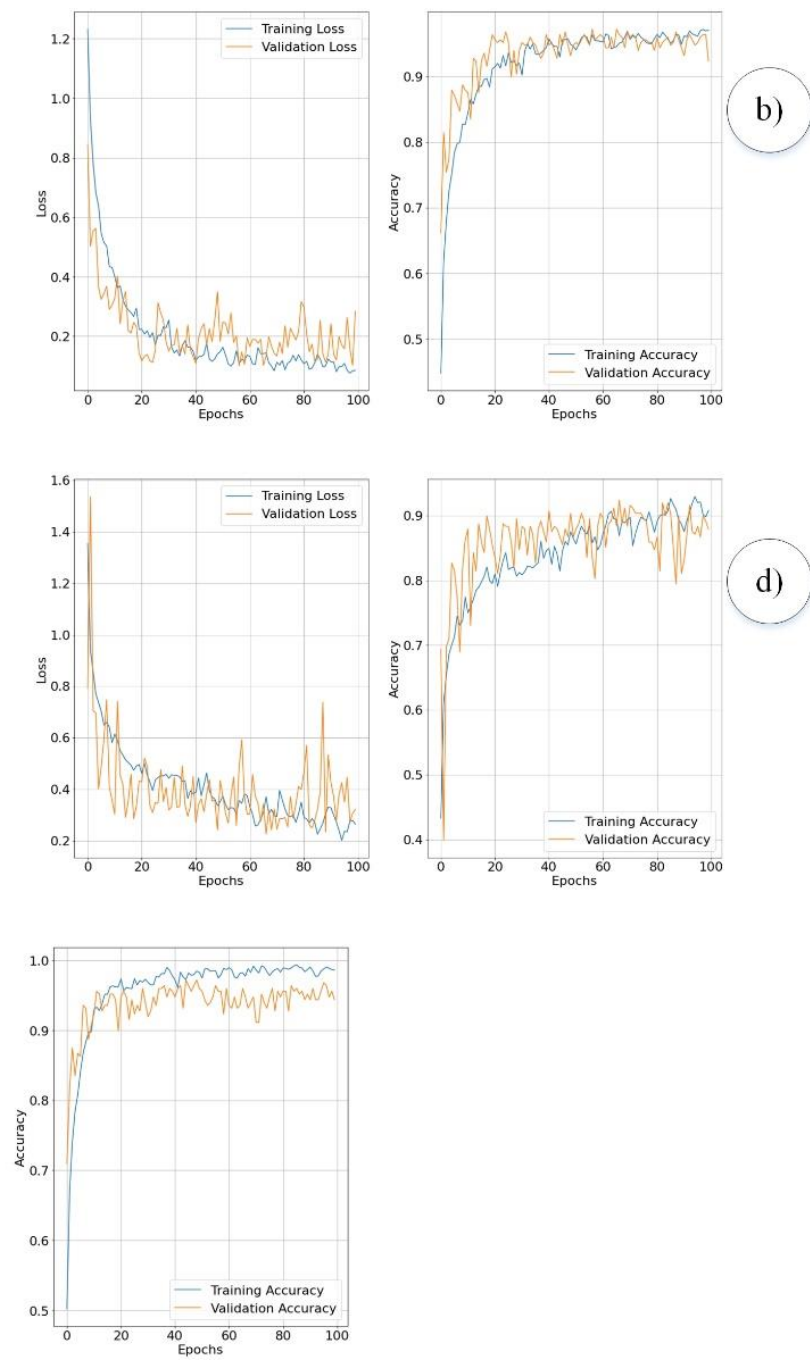

Figure 8. Training and validation losses and accuracies of the proposed CNN algorithm: According to the pre-trained network order used in the PFEM step; a) DenseNet121 plots, b) DenseNet201 plots, c) InceptionResNetv2 plots, d ) InceptionV3 plots, e) ResNet50V2 plots.

In the network settings, 100 epochs and 32 batch sizes have been adjusted. Figure 8 shows the loss and accuracy graphs of the training and validation obtained with each of the trained networks used in the PFEM method with the proposed CNN method. A comparison table was prepared with the models developed using the dataset published in 2020, which was used within the scope of this study. Looking at the prepared Table 4, accuracy was compared with two different studies published after the publication of the data set. The proposed approach yielded better accuracy than either of the two different studies. Bansal et al. (Bansal et al., 2021) reports in his study that he created a method using DenseNet121, EfficientNetB7 and EfficientNet NoisyStudent models. Using the specified pre-trained algorithms, it tried to detect plant disease quickly. Divakar et al. (Divakar et al., 2021) performed classification with the EfficientNetB7 method on a balanced data set obtained using the SMOTE method. He mentions that although different pre-trained models are used in the method, the most successful results are obtained in the EfficientNetB7 method. Both of these comparison methods used the data set used in this article. In this sense, the comparison chart given in Table 4 shows that the proposed method to classify leaf diseases is successful. 
Table 4. Comparison results with studies in the literature using the same dataset

\begin{tabular}{|l|l|l|l|}
\hline $\begin{array}{l}\text { Model } \\
\text { 2021) }\end{array}$ & Year & Method Accuracy \\
\hline $\begin{array}{l}\text { (Divakar et al., } \\
\text { 2021) }\end{array}$ & 2021 & $\begin{array}{l}\text { Deep Convolutional } \\
\text { Neural Network }\end{array}$ & 0.96 \\
\hline Proposed model & 2021 & $\begin{array}{l}\text { Convolution neural network } \\
\text { approach }\end{array}$ & 0.99 \\
\hline
\end{tabular}

\section{Conclusions}

Within the scope of this study, both validation and training features were extracted with the help of DenseNet121, DenseNet201, InceptionV3, ResNet50V2 and InceptionResNetV2 networks by preprocessing apple leaf diseases. A new classifier has been developed by giving the extracted feature values as input to the proposed CNN algorithm. As a result of the study, the accuracy results obtained from the ResNet50V2 network are higher than other network models. As a matter of fact, although the training period does not take long because the data set is small, the biggest disadvantage of pre-trained networks is the need for advanced computers. At this point, the operation cost can be reduced by selecting the best ones by feature extraction method from the extracted features.

\section{Conflict of Interest}

No conflict of interest was declared by the author.

\section{References}

Annabel, L. S. P., Annapoorani, T., \& Deepalakshmi, P. (2019). Machine Learning for Plant Leaf Disease Detection and Classification - A Review. 2019 International Conference on Communication and Signal Processing (ICCSP), 538-542. https://doi.org/10.1109/ICCSP.2019.8698004

Aurangzeb, K., Akmal, F., Khan, M. A., Sharif, M., \& Javed, M. Y. (2020). Advanced Machine Learning Algorithm Based System for Crops Leaf Diseases Recognition. 2020 6th Conference on Data Science and Machine Learning Applications (CDMA), 146151. https://doi.org/10.1109/CDMA47397.2020.00031

Bansal, P., Kumar, R., \& Kumar, S. (2021). Disease Detection in Apple Leaves Using Deep Convolutional Neural Network. In Agriculture (Vol. 11, Issue 7). https://doi.org/10.3390/agriculture11070617

Deng, X., Xu, D., Zeng, M., \& Qi, Y. (2019). Does Internet use help reduce rural cropland abandonment? Evidence from China. Land Use Policy, 89, 104243. https://doi.org/10.1016/j.landusepol.2019.104243

Divakar, S., Bhattacharjee, A., \& Priyadarshini, R. (2021). Smote-DL: A Deep Learning Based Plant Disease Detection Method. 2021 6th International Conference for Convergence in Technology (I2CT), 1-6. https://doi.org/10.1109/I2CT51068.2021.9417920

Dubey, S. R., \& Jalal, A. S. (2016). Apple disease classification using color, texture and shape features from images. Signal, Image and Video Processing, 10(5), 819-826. https://doi.org/10.1007/s11760-015-0821-1

Duralija, B., Putnik, P., Brdar, D., Bebek Markovinović, A., Zavadlav, S., Pateiro, M., Domínguez, R., Lorenzo, J. M., \& Bursać Kovačević, D. (2021). The Perspective of Croatian Old Apple Cultivars in Extensive Farming for the Production of Functional Foods. In Foods (Vol. 10, Issue 4). https://doi.org/10.3390/foods10040708

Ferentinos, K. P. (2018). Deep learning models for plant disease detection and diagnosis. Computers and Electronics in Agriculture, 145, 311-318. https://doi.org/10.1016/j.compag.2018.01.009

Gargade, A., \& Khandekar, S. A. (2019). A Review: Custard Apple Leaf Parameter Analysis and Leaf Disease Detection using Digital Image Processing. 2019 3rd International Conference on Computing Methodologies and Communication (ICCMC), 267-271. https://doi.org/10.1109/ICCMC.2019.8819867

Gobalakrishnan, N., Pradeep, K., Raman, C. J., Ali, L. J., \& Gopinath, M. P. (2020). A Systematic Review on Image Processing and Machine Learning Techniques for Detecting Plant Diseases. 2020 International Conference on Communication and Signal Processing (ICCSP), 465-468. https://doi.org/10.1109/ICCSP48568.2020.9182046

Gollapudi, S. (2019). Learn Computer Vision Using OpenCV. https://doi.org/10.1007/978-1-4842-4261-2

Han, H., Xiong, J., \& Zhao, K. (2021). Digital inclusion in social media marketing adoption: the role of product suitability in the agriculture sector. Information Systems and E-Business Management. https://doi.org/10.1007/s10257-021-00522-7

He, K., Zhang, X., Ren, S., \& Sun, J. (2016). Deep residual learning for image recognition. Proceedings of the IEEE Conference on Computer Vision and Pattern Recognition, 770-778.

Hoang, N.-D., \& Nguyễn Quốc, L. (2018). Metaheuristic Optimized Edge Detection for Recognition of Concrete Wall Cracks: A Comparative Study on the Performances of Roberts, Prewitt, Canny, and Sobel Algorithms. Advances in Civil Engineering, 2018, 1-16. https://doi.org/10.1155/2018/7163580

Hou, J., Huo, X., \& Yin, R. (2019). Does computer usage change farmers' production and consumption? Evidence from China. China Agricultural Economic Review, 11(2), 387-410. https://doi.org/10.1108/CAER-09-2016-0149

Huang, G., Liu, Z., Van Der Maaten, L., \& Weinberger, K. Q. (2017). Densely connected convolutional networks. Proceedings of the IEEE Conference on Computer Vision and Pattern Recognition, 4700-4708.

Iqbal, Z., Khan, M. A., Sharif, M., Shah, J. H., ur Rehman, M. H., \& Javed, K. (2018). An automated detection and classification of citrus plant diseases using image processing techniques: A review. Computers and Electronics in Agriculture, 153, 12-32. 
https://doi.org/10.1016/j.compag.2018.07.032

Ishaq, A., Sadiq, S., Umer, M., Ullah, S., Mirjalili, S., Rupapara, V., \& Nappi, M. (2021). Improving the Prediction of Heart Failure Patients' Survival Using SMOTE and Effective Data Mining Techniques. IEEE Access, 9, 39707-39716. https://doi.org/10.1109/ACCESS.2021.3064084

Khan, M. A., Akram, T., Sharif, M., \& Saba, T. (2020). Fruits diseases classification: exploiting a hierarchical framework for deep features fusion and selection. Multimedia Tools and Applications, 79(35), 25763-25783. https://doi.org/10.1007/s11042020-09244-3

Liang, Q., Xiang, S., Hu, Y., Coppola, G., Zhang, D., \& Sun, W. (2019). PD2SE-Net: Computer-assisted plant disease diagnosis and severity estimation network. Computers and Electronics in Agriculture, 157, 518-529. https://doi.org/10.1016/j.compag.2019.01.034

Mohanty, S. P., Hughes, D. P., \& Salathé, M. (2016). Using Deep Learning for Image-Based Plant Disease Detection. Frontiers in Plant Science, 7, 1419. https://doi.org/10.3389/fpls.2016.01419

Ni, A., Huang, L., \& Xiong, F. (2021). A new perspective of innovation-driven agricultural sustainable development: a case of China. IOP Conference Series: Earth and Environmental Science, 667, 12096. https://doi.org/10.1088/1755$1315 / 667 / 1 / 012096$

Prashar, K., Talwar, R., \& Kant, C. (2017). Robust Automatic Cotton Crop Disease Recognition (ACDR) Method using the Hybrid Feature Descriptor with SVM.

Shi, Y., Wang, X. F., Zhang, S. W., \& Zhang, C. L. (2015). PNN based crop disease recognition with leaf image features and meteorological data. International Journal of Agricultural and Biological Engineering, 8, 60-68. https://doi.org/10.3965/j.ijabe.20150804.1719

Shrivastava, G. (2021). Review on Emerging Trends in Detection of Plant Diseases using Image Processing with Machine Learning. International Journal of Computer Applications, 174. https://doi.org/10.5120/ijca2021920990

Singh, V., \& Misra, A. K. (2017). Detection of plant leaf diseases using image segmentation and soft computing techniques. Information Processing in Agriculture, 4(1), 41-49. https://doi.org/10.1016/j.inpa.2016.10.005

Sottocornola, G., Stella, F., \& Zanker, M. (2021). Counterfactual Contextual Multi-Armed Bandit: a Real-World Application to Diagnose Apple Diseases.

Sujatha, R., Chatterjee, J. M., Jhanjhi, N. Z., \& Brohi, S. N. (2021). Performance of deep learning vs machine learning in plant leaf disease detection. Microprocessors and Microsystems, 80, 103615. https://doi.org/10.1016/j.micpro.2020.103615

Szegedy, C., Ioffe, S., Vanhoucke, V., \& Alemi, A. A. (2017). Inception-v4, inception-resnet and the impact of residual connections on learning. Thirty-First AAAI Conference on Artificial Intelligence.

Szegedy, C., Vanhoucke, V., Ioffe, S., Shlens, J., \& Wojna, Z. B. (2016). Rethinking the Inception Architecture for Computer Vision. https://doi.org/10.1109/CVPR.2016.308

Tahir, M. Bin, Khan, M. A., Javed, K., Kadry, S., Zhang, Y.-D., Akram, T., \& Nazir, M. (2021). Recognition of Apple Leaf Diseases using Deep Learning and Variances-Controlled Features Reduction. Microprocessors and Microsystems, 104027. https://doi.org/10.1016/j.micpro.2021.104027

Thapa, R., Zhang, K., Snavely, N., Belongie, S., \& Khan, A. (2020). The Plant Pathology Challenge 2020 data set to classify foliar disease of apples. Applications in Plant Sciences, 8(9), e11390. https://doi.org/10.1002/aps3.11390

Tiwari, D., Ashish, M., Gangwar, N., Sharma, A., Patel, S., \& Bhardwaj, S. (2020). Potato Leaf Diseases Detection Using Deep Learning. https://doi.org/10.1109/ICICCS48265.2020.9121067

Turkoglu, M., Hanbay, D., \& Sengur, A. (2019). Multi-model LSTM-based convolutional neural networks for detection of apple diseases and pests. Journal of Ambient Intelligence and Humanized Computing. https://doi.org/10.1007/s12652-01901591-w

Wang, G., Sun, Y., \& Wang, J. (2017). Automatic Image-Based Plant Disease Severity Estimation Using Deep Learning. Computational Intelligence and Neuroscience, 2017, 2917536. https://doi.org/10.1155/2017/2917536

Zhang, S., Huang, W., \& Zhang, C. (2019). Three-channel convolutional neural networks for vegetable leaf disease recognition. Cognitive Systems Research, 53, 31-41. https://doi.org/10.1016/j.cogsys.2018.04.006

Zhu, X., Hu, R., Zhang, C., \& Shi, G. (2021). Does Internet use improve technical efficiency? Evidence from apple production in China. Technological Forecasting and Social Change, 166, 120662. https://doi.org/10.1016/j.techfore.2021.120662 